\title{
MODELLING OF DERIVATIVES PRICING USING METHODS OF SPECTRAL ANALYSIS
}

\author{
IVAN BURTNYAK, ANNA MALYTSKA
}

\begin{abstract}
In this article expands the method of finding the approximate price for a wide class of derivative financial instruments. Using the spectral theory of self-adjoint operators in Hilbert space and the wave theory of singular and regular perturbations, the analytical formula of the approximate asset price is established. Methods for calculating the approximate price of options using the tools of spectral analysis, singular and regular wave theory in the case of fast and slow factors are developed. Combining methods from the spectral theory of singular and regular perturbations, it is possible to estimate the price of derivative financial instruments as a schedule by eigenfunctions. The approximate value of securities and their rate of return are calculated. Applying the theory of Sturm-Liouville, Fredholm's alternative and analysis of singular and regular perturbations at different time scales have enabled us to obtain explicit formulas for the approximate value of securities and their yield on the basis of the development of their eigenfunctions and eigenvalues of self-adjoint operators using boundary value problems for singular and regular perturbations. The theorem of closeness estimates for bond prices approximation is proved. An algorithm for calculating the approximate price of derivatives and the accuracy of estimates has been developed, which allows to analyze and draw precautionary conclusions and suggestions to minimize the risks of pricing derivatives that arise in the stock market. A model for finding the value of derivatives corresponding to the dynamics of the stock market and the size of financial flows has been developed. This model allows you to find the prices of derivatives and their volatility, as well as minimize speculative changes in pricing, analyze the progress of stock market processes and take concrete steps to improve the situation to optimize financial strategies. The used methodology of European options pricing based on the study of volatility behavior and analysis of the yield of financial instruments allows to increase the accuracy of the forecast and make sound management strategic decisions by stock market participants.
\end{abstract}

Keywords: stock market, derivatives, spectral analysis, spectral theory, singular perturbation theory, regular perturbation theory.

JEL Classification: G11, G13, G32.

\section{INTRODUCTION}

Short-term interest rate dynamics models were considered in the paper by [12] for derivatives pricing. Significant contributions to the theory of rate of interest were made by [1], [8-10], namely: finding a credit spread of credit market instruments, calculating option prices for interest rates, determining the risk and derivatives' rate of return of the stock market financial instruments. The 
models developed by these scholars have their advantages and disadvantages, but each of them is used to increase the liquidity of financial markets. Applying more sophisticated models, despite their theoretical justification, leads to obtaining of complex multi-parameter functions of the yield curve, which results in significant errors in calculations.

Using spectral analysis, [5] applied a spectral theory of self-adjoint operators to different models, and to the Vasicek model, in particular. [11] considered the short-term interest rates described by the Vasicek model with stochastic volatility, depending on two factors, one of which is fast, and the other is slowly variable. In our paper, the spectral theory and the theory of singular and regular perturbations are applied to self-adjoint operators in Hilbert spaces that describe processes with multidimensional stochastic volatility having l-fast variables, $\mathrm{r}$-slowly variables, $l \geq 1, r \geq 1, l \in N, r \in N$. This theory is applied to the short-term interest rates described by the Vasicek model, in particular. The approximate value of securities and their yield are calculated. Applying the Sturm-Liouville theory, Fredholm alternatives, as well as analysis of singular and regular perturbations at different time scales, we obtained explicit formulas for convergence of bond prices and their yield. To obtain explicit formulas, we need to solve 21 Poisson equations.

\section{THEORETICAL BACKGROUND}

The purpose of the article is to establish bond indicative prices and their profitability by methods of spectral theory and theory of perturbations.

Let $(\mathrm{Q}, \mathrm{F}, \mathrm{P})$ be the probability space that supports a correlated Brownian motion $\left(\mathrm{W}^{x}, \mathrm{~W}^{y_{1}}, \ldots, \mathrm{W}^{y_{l}}, \mathrm{~W}^{z_{1}}, \ldots, \mathrm{W}^{z_{r}}\right)$ and an exponential random variable $\varepsilon \sim \operatorname{Exp}(1)$, which is independent of $\left(\mathrm{W}^{x}, \mathrm{~W}^{y_{1}}, \ldots, \mathrm{W}^{y_{l}}, \mathrm{~W}^{z_{1}}, \ldots, \mathrm{W}^{z_{r}}\right)$. We will assume that the economy with $(1+\mathrm{r}+1)$ factors is described by the homogeneous time and continuous Markov process $\chi=\left(X, Y_{1}, \ldots, Y_{1}, Z_{1}, \ldots, Z_{r}\right)$, which is defined in some state space $\mathrm{E}=I \times \mathrm{R}^{l} \times \mathrm{R}^{\mathrm{r}}$, where $\left(\mathrm{Y}_{1}, \ldots, \mathrm{Y}_{\mathrm{l}}\right) \in \mathrm{R}^{l},\left(\mathrm{Z}_{1}, \ldots, \mathrm{Z}_{\mathrm{r}}\right) \in \mathrm{R}^{\mathrm{r}}$. I is the interval at $\mathrm{R}$ with points $e_{1}$ and $e_{2}$, such that $-\infty<e_{1}<e_{2}<\infty$. We assume that $\chi$ has the beginning at $E$ and instantly disappears once $X$ goes beyond $I$. In particular, the dynamics of $\chi$ with physical measure $\mathbb{P}$ is as follows:

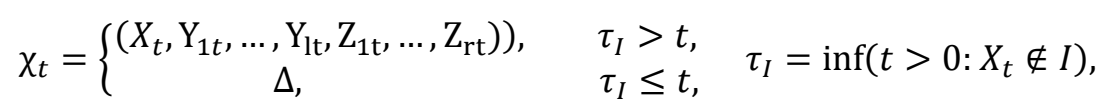

where $\left(X, Y_{1}, \ldots, Y_{1}, Z_{1}, \ldots, Z_{r}\right)$, are set

$$
\begin{aligned}
& d X_{t}=v\left(X_{t}\right) d t+a\left(X_{t}\right) f\left(\mathrm{Y}_{1 t}, \ldots, \mathrm{Y}_{\mathrm{lt}}, \mathrm{Z}_{1 \mathrm{t}}, \ldots, \mathrm{Z}_{\mathrm{rt}}\right) d W_{t}^{x} \\
& d Y_{j t}=\frac{1}{\epsilon_{j}} \alpha_{j}\left(Y_{j t}\right) d t+\frac{1}{\sqrt{\epsilon_{j}}} \beta_{j}\left(Y_{j t}\right) d W_{t}^{y_{j}}, j=\overline{1, l} . \\
& d Z_{i t}=\delta_{i} c_{i}\left(Z_{i t}\right) d t+\sqrt{\delta_{i}} g_{i}\left(Z_{i t}\right) d W_{t}^{z_{i}}, i=\overline{1, r} . \\
& d\left(W^{x}, W^{y_{j}}\right)_{t}=\rho_{x y_{j}} d t, j=\overline{1, l} . \\
& d\left(W^{x}, W^{z_{i}}\right)_{t}=\rho_{x z_{i}} d t, i=\overline{1, r} . \\
& d\left(W^{y_{j}}, W^{z_{i}}\right)_{t}=\rho_{y_{j} z_{i}} d t, j=\overline{1, l}, \quad i=\overline{1, r} . \\
& d\left(W^{y_{j}}, W^{y_{r}}\right)_{t}=\rho_{y_{j} y_{s}} d t, j=\overline{1, l}, \quad s=\overline{1, l} . \\
& d\left(W^{z_{i}}, W^{z_{k}}\right)_{t}=\rho_{z_{i} z_{k}} d t, i=\overline{1, n}, \quad k=\overline{1, r} . \\
& \left(X_{0}, \mathrm{Y}_{10}, \ldots, \mathrm{Y}_{10}, \mathrm{Z}_{10}, \ldots, \mathrm{Z}_{\mathrm{r} 0}\right)=\left(x, y_{10}, \ldots y_{l 0}, z_{10}, \ldots, z_{r 0}\right) \in E .
\end{aligned}
$$

where $\rho_{y_{j} y_{s}}=0, j \neq r, \rho_{z_{i} z_{k}}=0, i \neq k \rho_{x y_{j}}, \rho_{x z_{i}}, \rho_{y_{j} z_{i}}$, meet the conditions $\left|\rho_{x y_{j}}\right|,\left|\rho_{x z_{i}}\right|,\left|\rho_{y_{j} z_{i}}\right| \leq 1$, and correlation matrices of the form:

$$
\left(\begin{array}{ccc}
1 & \rho_{x y_{j}} & \rho_{x z_{i}} \\
\rho_{y_{j} x} & 1 & \rho_{y_{j} z_{i}} \\
\rho_{z_{i} x} & \rho_{z_{i} y_{j}} & 1
\end{array}\right)
$$

semipositively defined, that is $1+2 \rho_{x y_{j}} \rho_{x z_{i}} \rho_{y_{j} z_{i}}-\rho_{x y_{j}}{ }^{2}-\rho_{x z_{i}}{ }^{2}-\rho_{y_{j} z_{i}}{ }^{2} \geq 0, j=\overline{1, l}, \quad i=\overline{1, r}$.

Process $X$ may represent many economic phenomena and processes. 


\section{Research Objective, Methodology and Data}

For example, the reserve size, the index price and reliable short-term interest rates, etc. Even more broadly, $X$ is an external factor that characterizes the value of any of the above-mentioned processes. Physical measure $\mathbb{P}$ of process $X$ is understood as the process $X$, which has an instant drift $v\left(X_{t}\right)$ and stochastic volatility $a\left(X_{t}\right) f\left(\mathrm{Y}_{1 t}, \ldots, \mathrm{Y}_{\mathrm{lt}}, \mathrm{Z}_{1 \mathrm{t}}, \ldots, \mathrm{Z}_{\mathrm{nt}}\right)>0$, which contains both components: local $a\left(X_{t}\right)$ and nonlocal $f\left(\mathrm{Y}_{1 t}, \ldots, \mathrm{Y}_{\mathrm{lt}}, \mathrm{Z}_{1 \mathrm{t}}, \ldots, \mathrm{Z}_{\mathrm{nt}}\right)$. Note that infinitesimal generators (infinitized) for $Y_{j}$ and $Z_{i}$ have the form $\forall i, j$

$$
\mathfrak{\Omega}_{Y_{j}}^{\epsilon_{j}}=\frac{1}{\epsilon_{j}}\left(\frac{1}{2} \beta_{j}^{2}\left(y_{j}\right) \partial_{y_{j} y_{j}}^{2}+\alpha_{j}\left(y_{j}\right) \partial_{y_{j}}\right), \mathfrak{Q}_{z_{i}}^{\delta_{i}}=\delta_{i}\left(\frac{1}{2} g_{i}^{2}\left(z_{i}\right) \partial_{z_{i} z_{i}}^{2}+c_{i}\left(z_{i}\right) \partial_{z_{i}}\right),
$$

are characterized by the measures $\frac{1}{\epsilon_{j}}$ and $\delta_{i}$, respectively. Thus, $\mathrm{Y}_{1}, \ldots, \mathrm{Y}_{\mathrm{l}}$ and $\mathrm{Z}_{1}, \ldots, \mathrm{Z}_{\mathrm{n}}$ have an internal time scale $\epsilon_{j}>0$ and $\frac{1}{\delta_{i}}>0$. We consider $\epsilon_{j}<<1$ and $\delta_{i}<<1$, so that the internal time scale $Y_{j}$ is small, and the internal time scale $Z_{i}$ is large. Consequently, $Y_{j}, j=\overline{1, l}$, are fast variables, and $Z_{i}, i=\overline{1, n}$ are slowly variables. Note that $\mathfrak{R}_{Y_{j}}^{\epsilon_{j}}$ and $\mathfrak{R}_{Z_{i}}^{\delta_{i}}$ have the form [6]

$$
L=\frac{1}{2} a^{2}(x) \partial_{x x}^{2}+b(x) \partial_{x}-k(x), x \in\left(e_{1}, e_{2}\right), 3 k(x)=0
$$

for all $x \in I$, are always self-adjoint in the Hilbert space $H=L^{2}(I, m)$, where $I \in R$ is the interval with the points $e_{1}$ and $e_{2}$ and $m$ is the diffusion density rate. $\operatorname{Dom}(\mathfrak{L})\left\{f \in L^{2}(I, \mathfrak{m}): f, \partial_{x} f \in A C_{\text {loc }}(I), \mathfrak{L} f L^{2}(I, \mathfrak{m}), B C\right.$ s on $e_{1}$ and $\left.e_{2}\right\}$ where $A C_{\text {loc }}(I)$ is the space of functions which are absolutely continuous on each compact subinterval $I$ [3]. The boundary conditions for $e_{1}$ and $e_{2}$ are applied on the output, input, and regular bounds.

We will evaluate the derivatives with payoff at time $t>0$, which may depend on the trajectory $X$. In particular, we will consider the forms of payoff:

$$
\text { Payoff }=H\left(X_{t}\right) \mathbb{I}_{(\tau>t)},
$$

where $\tau$ is a random moment of time during which there is a failure to make a payment of premium. Since we are interested in the derivatives estimation, we must determine the dynamics $\left(\mathrm{X}, \mathrm{Y}_{1}, \ldots, \mathrm{Y}_{1}, \mathrm{Z}_{1}, \ldots, \mathrm{Z}_{\mathrm{r}}\right)$, under the evaluation of the degree of neutral risk, which we denote as $\widetilde{\mathbb{P}}$. We have the following dynamics [7]:

where

$$
\begin{aligned}
& d X_{t}=\left(b\left(X_{t}\right)-a\left(X_{t}\right) f\left(\mathrm{Y}_{1 t}, \ldots, \mathrm{Y}_{\mathrm{lt}}, \mathrm{Z}_{1 \mathrm{t}}, \ldots, \mathrm{Z}_{\mathrm{rt}}\right) \Omega\left(\mathrm{Y}_{1 t}, \ldots, \mathrm{Y}_{\mathrm{lt}}, \mathrm{Z}_{1 \mathrm{t}}, \ldots, \mathrm{Z}_{\mathrm{rt}}\right)\right) d t \\
& +a\left(X_{t}\right) f\left(\mathrm{Y}_{1 t}, \ldots, \mathrm{Y}_{\mathrm{lt}}, \mathrm{Z}_{1 \mathrm{t}}, \ldots, \mathrm{Z}_{\mathrm{rt}}\right) d \widetilde{W}_{t}^{x}, \\
& d Y_{j t}=\left(\frac{1}{\epsilon_{j}} \alpha_{j}\left(Y_{j t}\right)-\frac{1}{\sqrt{\epsilon_{j}}} \beta_{j}\left(Y_{j t}\right) \Lambda_{j}\left(\mathrm{Y}_{1 t}, \ldots, \mathrm{Y}_{\mathrm{lt}}, \mathrm{Z}_{1 \mathrm{t}}, \ldots, \mathrm{Z}_{\mathrm{rt}}\right)\right) d t+\frac{1}{\sqrt{\epsilon_{j}}} \beta_{j}\left(Y_{j t}\right) d \widetilde{W}_{t}^{y_{j}}, \\
& d Z_{i t}=\left(\delta_{i} c_{i}\left(Z_{i t}\right)-\sqrt{\delta_{i}} g_{i}\left(Z_{i t}\right) \Gamma_{i}\left(\mathrm{Y}_{1 t}, \ldots, \mathrm{Y}_{\mathrm{lt}}, \mathrm{Z}_{1 \mathrm{t}}, \ldots, \mathrm{Z}_{\mathrm{rt}}\right)\right) d t+\sqrt{\delta_{i}} g_{i}\left(Z_{i t}\right) d \widetilde{W}_{t}^{Z_{i}}, \\
& d\left\langle\widetilde{W}^{x}, \widetilde{W}^{y_{j}}\right\rangle_{t}=\rho_{x y_{j}} d t, j=\overline{1, l} \text {. } \\
& d\left\langle\widetilde{W}^{x}, \widetilde{W}^{z_{i}}\right\rangle_{t}=\rho_{x z_{i}} d t, i=\overline{1, r} \\
& d\left\langle\widetilde{W}^{y_{j}}, \widetilde{W}^{z_{i}}\right\rangle_{t}=\rho_{y_{j} z_{i}} d t, j=\overline{1, l}, \quad i=\overline{1, r} . \\
& d\left\langle\widetilde{W}^{y_{j}}, \widetilde{W}^{y_{s}}\right\rangle_{t}=\rho_{y_{j} y_{s}} d t, j=\overline{1, l}, s=\overline{1, l} . \\
& d\left\langle\widetilde{W}^{z_{i}}, \widetilde{W}^{z_{k}}\right\rangle_{t}=\rho_{z_{i} z_{k}} d t, i=\overline{1, n}, \quad k=\overline{1, n} . \\
& \left(X_{0}, \mathrm{Y}_{10}, \ldots, \mathrm{Y}_{10}, \mathrm{Z}_{10}, \ldots, \mathrm{Z}_{\mathrm{r} 0}\right)=\left(x, y_{10}, \ldots y_{l 0}, z_{10}, \ldots, z_{r 0}\right) \in E \text {, }
\end{aligned}
$$

$$
\begin{aligned}
& d \widetilde{W}_{t}^{x}:=d W_{t}^{x}+\left(\frac{v\left(X_{t}\right)-b\left(X_{t}\right)}{a\left(X_{t}\right) f\left(\mathrm{Y}_{1 t}, \ldots, \mathrm{Y}_{\mathrm{lt}}, \mathrm{Z}_{1 \mathrm{t}}, \ldots, \mathrm{Z}_{\mathrm{nt}}\right)}+\Omega\left(\mathrm{Y}_{1 t}, \ldots, \mathrm{Y}_{\mathrm{lt}}, \mathrm{Z}_{1 \mathrm{t}}, \ldots, \mathrm{Z}_{\mathrm{rt}}\right)\right) d t \\
& d \widetilde{W}_{t}^{y_{j}}:=d W_{t}^{y_{j}}+\Lambda_{j}\left(\mathrm{Y}_{1 t}, \ldots, \mathrm{Y}_{\mathrm{lt}}, \mathrm{Z}_{1 \mathrm{t}}, \ldots, \mathrm{Z}_{\mathrm{rt}}\right) d t \\
& d \widetilde{W}_{t}^{Z_{i}}:=d W_{t}^{Z_{i}}+\Gamma_{i}\left(\mathrm{Y}_{1 t}, \ldots, \mathrm{Y}_{\mathrm{lt}}, \mathrm{Z}_{1 \mathrm{t}}, \ldots, \mathrm{Z}_{\mathrm{rt}}\right) d t
\end{aligned}
$$


where $\rho_{y_{j} y_{s}}=0, j \neq s, \rho_{z_{i} z_{k}}=0, i \neq k$.

We establish such conditions so that the system (1) has the only strong solution.

Random time $\tau$ is the time of the derivative asset. In our case, default can occur in one of two ways:

1) when $X$ fall outside the interval $I$,

2) at random time $\tau_{h}$, which is managed by the risk level $h\left(X_{t}\right) \geq 0$.

This can be expressed as follows:

$$
\begin{gathered}
\tau=\tau_{I}^{\wedge} \tau_{h}, \\
\tau_{h}=\inf \left\{t \geq 0: \int_{0}^{t} h\left(X_{s}\right) d s \geq \varepsilon\left(X, \mathrm{Y}_{1}, \ldots, \mathrm{Y}_{1}, \mathrm{Z}_{1}, \ldots, \mathrm{Z}_{\mathrm{n}}\right)\right\}, \\
\varepsilon \sim \operatorname{Exp}(1) \Perp .
\end{gathered}
$$

Note that the random variable $\varepsilon$ is independent of $\left(X, \mathrm{Y}_{1}, \ldots, \mathrm{Y}_{1}, \mathrm{Z}_{1}, \ldots, \mathrm{Z}_{\mathrm{n}}\right)$.

To track $\tau_{h}$, we use the process indicator: $D_{t}=\mathbb{I}_{\left\{t \geq \tau_{h}\right\}}$, where $\mathbb{D}=\left\{\mathfrak{D}_{t}, t \geq 0\right\}$, is a filter generated by $D$ and $\mathbb{F}=\left\{\mathfrak{f}_{t}, t \geq 0\right\}$ is filter's generator $\left(W^{x}, W^{y_{1}}, \ldots W^{y_{l}}, W^{z_{1}}, \ldots, W^{z_{n}}\right)$. We use the filtering $\mathbb{G}=\left\{\mathfrak{G}_{t}, t \geq 0\right\}$, where $\mathfrak{G}_{t}=\mathfrak{F}_{t} \mathrm{VD}_{t}$. Note that $\left(X, \mathrm{Y}_{1}, \ldots, \mathrm{Y}_{\mathrm{l}}, \mathrm{Z}_{1}, \ldots, \mathrm{Z}_{\mathrm{n}}\right)$ are applied to $\mathbb{G}$ and $\tau$ is a stopping time $\left(\{\{\tau \leq t\}\} \in \mathfrak{G}_{t}\right.$ for all $\left.t \geq 0\right)$.

We will evaluate the derivative asset of some payoff (payment) using the neutral pricing risk and Markovian chain $X$, the price $u^{\bar{\epsilon}, \overline{\delta^{\prime}}}\left(t, x, y_{1}, \ldots y_{l}, z_{1}, \ldots, z_{r}\right)$ of some derivative assets at the initial moment of time has the form:

$$
u^{\bar{\epsilon}, \overline{\delta^{\prime}}}\left(t, x, y_{1}, \ldots y_{l}, z_{1}, \ldots, z_{r}\right)=\widetilde{\mathbb{E}}_{x, y_{1}, \ldots y_{l}, z_{1}, \ldots, z_{r}}\left[\exp \left(-\int_{0}^{t} r\left(X_{S}\right) d s\right) H\left(X_{t} \mathbb{I}_{\{t>\tau\}}\right)\right],
$$

where $\bar{\epsilon}=\left(\epsilon_{1}, \ldots, \epsilon_{l}\right), \overline{\delta^{\prime}}=\left(\delta_{1}, \ldots, \delta_{r}\right)$, and $\left(x, y_{1}, \ldots y_{l}, z_{1}, \ldots, z_{r}\right) \in E$ is a starting point of the process $\left(X, \mathrm{Y}_{1}, \ldots, \mathrm{Y}_{1}, \mathrm{Z}_{1}, \ldots, \mathrm{Z}_{\mathrm{r}}\right)$. By Feynmann-Kac formulas, we can show that $u^{\bar{\epsilon}} \overline{\delta^{\prime}}\left(t, x, y_{1}, \ldots y_{l}, z_{1}, \ldots, z_{r}\right)$ satisfies the following Cauchy problem [3]:

$$
\begin{aligned}
& \left(-\partial_{t}+\mathfrak{Q}^{\bar{\epsilon}} \overline{\delta^{\prime}}\right) u^{\bar{\epsilon}, \overline{\delta^{\prime}}}=0, \quad\left(y_{1}, \ldots y_{l}, z_{1}, \ldots, z_{r}\right) \in E, t \in \mathbb{R}^{+}, \\
& u^{\bar{\epsilon}, \overline{\delta^{\prime}}}\left(0, x, y_{1}, \ldots y_{l}, z_{1}, \ldots, z_{r}\right)=H(x),
\end{aligned}
$$

where the operator $\mathfrak{Q}^{\bar{\epsilon}} \overline{\delta^{\prime}}$ has the form:

$$
\begin{aligned}
& \mathfrak{Q}^{\bar{\epsilon}, \overline{\delta^{\prime}}}=\sum_{j=1}^{l} \frac{1}{\epsilon_{j}} \mathfrak{L}_{0 j}+\sum_{j=1}^{l} \frac{1}{\sqrt{\epsilon_{j}}} \mathfrak{Q}_{1 j}+\mathfrak{Q}_{2 j}+\sum_{i, j} \sqrt{\frac{\delta_{i}}{\epsilon_{j}}} \mathfrak{M}_{3 i j}+\sum_{i} \sqrt{\delta_{i}} \mathfrak{M}_{1 i}+\sum_{i} \delta_{i} \mathfrak{M}_{2 i}, \\
& \mathfrak{L}_{0 j}=\frac{1}{2} \beta_{j}^{2}\left(y_{j}\right) \partial_{y_{j} y_{j}}^{2}+\alpha_{j}\left(y_{j}\right) \partial_{y_{j}}, \quad j=\overline{1, l} . \\
& \mathfrak{Q}_{1 j}=\beta_{j}\left(y_{j}\right)\left(\rho_{x y_{j}} a(x) f\left(y_{1}, \ldots y_{l}, z_{1}, \ldots, z_{r}\right) \partial_{x}-\Lambda_{j}\left(y_{1}, \ldots y_{l}, z_{1}, \ldots, z_{r}\right)\right) \partial_{y_{j}}, \\
& \mathfrak{Q}_{2 j}=\frac{1}{2} a^{2}(x) f^{2}\left(y_{1}, \ldots y_{l}, z_{1}, \ldots, z_{r}\right) \partial_{x x}^{2}+\left(b(x)-a(x) \Omega\left(y_{1}, \ldots y_{l}, z_{1}, \ldots, z_{r}\right) f\left(y_{1}, \ldots y_{l}, z_{1}, \ldots, z_{r}\right)\right) \partial_{x}-k(x), \\
& \mathfrak{M}_{3 i j}=\rho_{x z_{i}} \beta_{j}\left(y_{j}\right) g_{i}\left(z_{i}\right) \partial_{y_{j} z_{i}}^{2}, \\
& \mathfrak{M}_{1 i}=g_{i}\left(z_{i}\right)\left(\rho_{x z_{i}} a(x) f\left(y_{1}, \ldots y_{l}, z_{1}, \ldots, z_{r}\right) \partial_{x}-\Gamma_{i}\left(y_{1}, \ldots y_{l}, z_{1}, \ldots, z_{r}\right)\right) \partial_{z_{i}}, \\
& \mathfrak{M}_{2 i}=\frac{1}{2} g_{i}^{2}\left(z_{i}\right) \partial_{z_{i} z_{i}}^{2}+c_{i}\left(z_{i}\right) \partial_{z_{i}}, k(x)=r(x)+h(x), \quad \mathfrak{L}_{0 j}=\mathfrak{L}_{Y_{j}}^{1} .
\end{aligned}
$$

We assume that the diffusion with the infinitesimal generator $\mathfrak{L}_{Y_{j}}^{1}$ has an invariant distribution $\Pi$ with density $\pi_{j}\left(y_{j}\right)$.

$$
\pi_{j}\left(y_{j}\right)=\frac{2}{\beta_{j}^{2}\left(y_{j}\right)} \exp \left\{\int_{y_{j 0}}^{y_{j}} \frac{2 \alpha_{j}(\theta)}{\beta_{j}^{2}(\theta)} d \theta\right\}, \quad \forall j=\overline{1, l}
$$

Besides the initial condition (3), the function $u^{\bar{\epsilon}, \overline{\delta^{\prime}}}\left(t, x, y_{1}, \ldots y_{l}, z_{1}, \ldots, z_{r}\right)$ must meet boundary conditions at the points of $e_{1}$ and $e_{2}$ of the interval $I$. The boundary conditions at points $e_{1}$ and $e_{2}$ 
belong to the domain $\mathfrak{Q}^{\bar{\epsilon}} \overline{\delta^{\prime}}$ and will depend on the nature of process $X$ on the points of $I$ and are classified as natural, output, input or regular [1]. The Cauchy problem (2)-(3) for $\left(f, \alpha_{1}, \ldots \alpha_{l}, \beta_{1}, \ldots, \beta_{r}, \Lambda_{1}, \ldots \Lambda_{l}, c_{1}, \ldots, c_{r}, g_{1}, \ldots g_{r}, \Gamma_{1}, \ldots, \Gamma_{r}\right)$ has no analytical solution. However, for fixed $\overline{\delta^{\prime}}$, the conditions containing $\bar{\epsilon}$ and are arbitrarily deviated in the $\bar{\epsilon}$-axis, which causes singular perturbations. For a fixed $\epsilon_{j}$ condition containing $\delta_{i}$ are small for some small $\overline{\delta^{\prime}}$-axis, which causes regular perturbations. Thus, the $\bar{\epsilon}$-axis and $\overline{\delta^{\prime}}$-axis yields the combined singular-regular perturbation of $\mathcal{O}(1)$ of the operator $\mathfrak{S}_{2}$. To find the asymptotic solution of the Cauchy problem (2)-(3), we develop $u^{\bar{\epsilon}} \overline{\delta^{\prime}}$ in orders $\sqrt{\epsilon_{j}}$ and $\sqrt{\delta_{i}}[2]$ :

$$
u^{\bar{\epsilon} \overline{\delta^{\prime}}}=\sum_{i_{1} \geq 0} \ldots \sum_{i_{l} \geq 0} \sum_{j_{1} \geq 0} \ldots \sum_{j_{r} \geq 0}{\sqrt{\epsilon_{1}}}^{j_{1}} \ldots{\sqrt{\epsilon_{l}}}^{j_{l}}{\sqrt{\delta_{1}}}^{i_{1}} \ldots{\sqrt{\delta_{r}}}^{i_{r}} u_{j_{1}, \ldots, j_{n}, i_{1}, \ldots i_{l}},
$$

where $\sum_{i_{1} \geq 0} \ldots \sum_{i_{l} \geq 0} \sum_{j_{1} \geq 0} \ldots \sum_{j_{r} \geq 0}{\sqrt{\epsilon_{1}}}^{j_{1}} \ldots{\sqrt{\epsilon_{l}}}^{j_{l}}{\sqrt{\delta_{1}}}^{i_{1}} \ldots{\sqrt{\delta_{r}}}^{i_{r}} u_{j_{1}, \ldots, j_{r}, i_{1}, \ldots i_{l}}=$

$$
\lim _{i \rightarrow \infty} \sum_{i_{1} \geq 0}^{m_{1}} \ldots \sum_{i_{l} \geq 0}^{m_{l} \geq 0} \sum_{j_{1} \geq 0}^{m_{l+1} \geq 0} \ldots \sum_{j_{n} \geq 0}^{m_{l+r}}{\sqrt{\epsilon_{1}}}^{j_{1}} \ldots{\sqrt{\epsilon_{l}}}^{j_{l}}{\sqrt{\delta_{1}}}^{i_{1}} \ldots \sqrt{\delta_{r}} u_{j_{1}, \ldots, j_{r}, i_{1}, \ldots i_{l}}, \quad m_{1} \rightarrow \infty, \ldots, m_{l+r} \rightarrow \infty .
$$

The approximate price is calculated

$$
u^{\bar{\epsilon}, \overline{\delta^{\prime}}} \approx u_{\overline{0}, \overline{0^{\prime}}}+\sum_{j=1}^{l} \sqrt{\epsilon_{j}} u_{\overline{1_{j}, \overline{0}^{\prime}}}+\sum_{i=1}^{r} \sqrt{\delta_{i}} u_{\overline{0}, \overline{1_{i}^{\prime}}}
$$

The choice of development in half-integer orders $\epsilon_{j}$ and $\delta_{i}$ are natural for $\mathfrak{Q}^{\bar{\epsilon}} \overline{\delta^{\prime}}$.

By conducting an analysis of singular perturbations at the corresponding levels, we obtain that $u_{\overline{0}, \overline{0^{\prime}}}, u_{\overline{1} j, 0^{\prime}}, u_{\overline{0}, \overline{1_{i}^{\prime}}}$ do not depend on $y_{1}, \ldots y_{l}$. The basic findings of the asymptotic analysis are given using the following formulas [4]

$$
\begin{aligned}
& \mathcal{O}(1): \sum_{j=1} \mathfrak{L}_{0 j} u_{\overline{2_{j}, \overline{0}^{\prime}}}+\left(-\partial_{t}+\left\langle\mathfrak{\Omega}_{2}\right\rangle\right) u_{\overline{0}, \overline{0^{\prime}}}=0, u_{\overline{0,0^{\prime}}}\left(0, x, z_{1}, \ldots, z_{r}\right)=H(x), \\
& \mathcal{O}\left(\sqrt{\epsilon_{j}}\right): \mathfrak{Q}_{0 j} u_{\overline{3}_{j}, \overline{0}^{\prime}}+\mathfrak{Q}_{1 j} u_{\overline{2}_{j}, \overline{0^{\prime}}}+\left(-\partial_{t}+\left\langle\mathfrak{R}_{2}\right\rangle\right) u_{\overline{1}_{j}, \overline{0}^{\prime}}+\sum_{k \neq j} \mathfrak{R}_{1 k} u_{\overline{1}_{k j}, \overline{0^{\prime}}}+\sum_{i \neq j} \mathfrak{R}_{1 i} \\
& =\mathcal{A}_{j} u_{\overline{0}, \overline{0^{\prime}}}, \quad u_{\overline{1}_{j}, \overline{0}^{\prime}}\left(0, x, z_{1}, \ldots, z_{n}\right)=0 \text {, } \\
& \overline{1}_{k j}=(\underbrace{0, \ldots 1}_{k} \underbrace{0,1,0, \ldots 0}_{j}) \text {. }
\end{aligned}
$$

According to the analysis of regular perturbations we have

$$
\mathcal{O}\left(\sqrt{\delta_{i}}\right):\left(-\partial_{t}+\left\langle\mathfrak{E}_{2}\right\rangle\right) u_{\overline{0}, \overline{1_{i}^{\prime}}}=\mathcal{B}_{i} \partial_{z_{i}} u_{\overline{0}, \overline{0^{\prime \prime}}}, u_{\overline{0}, \overline{1_{i}^{\prime}}}\left(0, x, z_{1}, \ldots, z_{r}\right)=0, i=\overline{1, r}
$$

Operators $\left\langle\mathfrak{R}_{2}\right\rangle, \mathcal{A}_{j}, \mathcal{B}_{i}$ and $\partial_{z_{i}}$ are defined by the formulas

$\left\langle\mathfrak{\Omega}_{2}\right\rangle=\frac{1}{2} \bar{\sigma}^{2} a^{2}(x) \partial_{x x}^{2}+(b(x)-\overline{f \Omega} a(x)) \partial_{x}-k(x), \quad x \in\left(e_{1}, e_{2}\right)$,

$\mathcal{A}_{j}=-v_{3 j} a(x) \partial_{x} a^{2}(x) \partial_{x x}^{2}-v_{2 j} a^{2}(x) \partial_{x x}^{2}-\mathcal{U}_{2 j} a(x) \partial_{x} a(x) \partial_{x}-u_{1 j} a(x) \partial_{x}$,

$\mathcal{B}_{i}=-v_{1 i} a(x) \partial_{x}-v_{0 i}$ and $\partial_{z_{i}}=\partial_{z_{i}} \bar{\sigma} \partial_{\bar{\sigma}}+\overline{f \Omega^{\prime}} \partial_{\bar{f} \Omega}, \quad v_{1 i}:=g_{i} \rho_{x z_{i}}\langle f\rangle, \quad v_{0}=g_{i}\left\langle\Gamma_{i}\right\rangle, \forall i=\overline{1, n}$ and norm function

$$
\begin{aligned}
& \langle X\rangle_{j}:=\int \mathcal{X}\left(y_{1}, \ldots y_{l}\right) \pi_{j}\left(y_{j}\right) d y_{j}, \quad \forall j=\overline{1, l}, \\
& \langle X\rangle_{1,2}=\int_{R^{2}} x\left(y_{1}, \ldots y_{l}\right) \pi_{1}\left(y_{1}\right) \pi_{2}\left(y_{2}\right) d y_{1} d y_{2}, \ldots\langle X\rangle_{l-1, l}= \\
& \int_{R^{l}} x\left(y_{1}, \ldots y_{l}\right) \pi_{1}\left(y_{1}\right) \ldots \pi_{l}\left(y_{l}\right) d y_{1} \ldots d y_{l},\langle X\rangle_{l-1, l}=\langle X\rangle,\langle f \Omega\rangle:=\overline{f \Omega},\left\langle f^{2}\right\rangle=\bar{\sigma}^{2} .
\end{aligned}
$$

We find solutions to the equations (4)-(6) on the basis of eigenfunctions, eigenvalues of the operator $\left\langle\Omega_{2}\right\rangle$, each of which meets a corresponding Poisson equation

$$
\begin{aligned}
& \mathfrak{L}_{01} \varphi_{1}=f^{2}-\left\langle f^{2}\right\rangle_{1}, \mathfrak{L}_{02} \varphi_{2}=\left\langle f^{2}\right\rangle_{1}-\left\langle f^{2}\right\rangle_{1,2}, \ldots, \mathfrak{Q}_{0 l} \varphi_{l}=\left\langle f^{2}\right\rangle_{l-2, l-1}-\left\langle f^{2}\right\rangle_{l-1, l}, \\
& \mathfrak{L}_{01} \eta_{1}=f \Omega-\langle f \Omega\rangle_{1}, \ldots, \mathfrak{L}_{0 j} \eta_{j}=\langle f \Omega\rangle_{j-2, j-1}-\langle f \Omega\rangle_{j-1, j}, \ldots, \mathfrak{L}_{0 l} \eta_{l}=\langle f \Omega\rangle_{l-2, l-1}-\langle f \Omega\rangle_{l-1, l} .
\end{aligned}
$$


Theorem 1: Assume that we can solve the following equation to find an eigenvalue:

$$
-\left\langle\mathfrak{R}_{2}\right\rangle \psi_{n}=\lambda_{n} \psi_{n}, \quad \psi_{n} \in \operatorname{dom}\left(\left\langle\mathfrak{I}_{2}\right\rangle\right),
$$

and also that $H \in \mathcal{H}$. Then the solution $u_{\overline{0}, \overline{0^{\prime}}}$ has the form:

$$
u_{\overline{0}, \overline{0^{\prime}}}=\sum_{n=1}^{\infty} c_{n} \psi_{n} T_{n}, \quad c_{n}=\left(\psi_{n}, H\right), \quad T_{n}=e^{-t \lambda_{n}} .
$$

Theorem 2: Let $c_{n}, \psi_{n}, T_{n}$ be described using Theorem 1. We define

$$
\mathcal{A}_{j k, n}:=\left(\psi_{k}, \mathcal{A}_{j} \psi_{n}\right), U_{k, n}:=\frac{T_{k}-T_{n}}{\lambda_{k}-\lambda_{n}} .
$$

Then the solution $u_{\overline{1}_{j}, \overline{0}^{\prime}}$ of equation (5) has the form:

$$
u_{\overline{1}_{j}, \overline{0}^{\prime}}=\sum_{n} \sum_{k \neq n} c_{n} A_{j k, n} \psi_{k} U_{k, n}-\sum_{n} c_{n} \mathcal{A}_{j n, n} \psi_{n} t T_{n} .
$$

Note that $u_{\overline{1}_{j}, \overline{0}^{\prime}}$ is linear in the parameter group $\left(\vartheta_{3 j}, \vartheta_{2 j}, u_{2 j}, u_{1 j}\right)$.

Theorem 3: Let $c_{n}, \psi_{n}$ and $T_{n}$ be defined with Theorem 1, and $U_{k, n}$ with Theorem 2, we have $\widetilde{\mathcal{B}}_{i k, n}:=\left(\psi_{k}, \mathcal{B}_{i} \partial_{Z_{i}} \psi_{n}\right), \quad \mathcal{B}_{i k, n}:=\left(\psi_{k}, \mathcal{B}_{i} \psi_{n}\right), \quad V_{i k, n}:=\frac{T_{k}-T_{n}}{\left(\lambda_{k}-\lambda_{n}\right)^{2}}+\frac{t T_{n}}{\lambda_{k}-\lambda_{n}}$.

Then the solution $u_{\overline{0}, \overline{1}_{i}^{\prime}}$ has the form:

$$
\begin{aligned}
u_{\overline{0}, \overline{1_{i}^{\prime}}}= & \sum_{n} \sum_{k \neq n} c_{n} \widetilde{\mathcal{B}}_{i k, n} \psi_{k} U_{i k, n}-\sum_{n} c_{n} \widetilde{\mathcal{B}}_{i n, n} \psi_{n} t T_{n} \\
& +\sum_{n} \sum_{k \neq n}\left(\partial_{Z_{i}} c_{n}\right) \mathcal{B}_{i k, n} \psi_{k} U_{i k, n}-\sum_{n}\left(\partial_{Z_{i}} c_{n}\right) \mathcal{B}_{i n, n} \psi_{n} t T_{n} \\
& +\sum_{n} \sum_{k \neq n} c_{n} \mathcal{B}_{i k, n} \psi_{k}\left(\partial_{Z_{i}} \lambda_{n}\right) V_{i k, n}-\sum_{n} c_{n} \mathcal{B}_{i n, n} \psi_{n}\left(\partial_{Z_{i}} \lambda_{n}\right) \frac{1}{2} t^{2} T_{n} .
\end{aligned}
$$

We draw attention to the fact that $u_{\overline{0}, 1_{i}^{\prime}}$ is linear in $\left(v_{1 i} \bar{\sigma}^{\prime}, v_{1 i} \overline{f \Omega^{\prime}}, v_{0 i} \bar{\sigma}^{\prime}, v_{0 i} \overline{f \Omega^{\prime}}\right)$.

Having obtained the approximate solution $u^{\bar{\epsilon}, \overline{\delta^{\prime}}} \approx u_{\overline{0}, \overline{0^{\prime}}}+\sum_{j=1}^{l} \sqrt{\epsilon_{j}} u_{\overline{j_{j}, 0^{\prime}}}+\sum_{i=1}^{n} \sqrt{\delta_{i}} u_{\overline{0}, \overline{1_{i}^{\prime}}}$ for the derivative asset pricing.

\section{RESUlTS AND DiscusSiON}

For a more exact result we assume that the Payoff function $H(x)$ and its derivative are smooth and limited functions. Thus, we limit our derivative analysis to a smooth and limited payoff; in this case, the closeness estimates is based on the following theorem:

Theorem 4: For the fixed $\left(t, x, y_{1}, \ldots, y_{l}, z_{1}, \ldots, z_{r}\right)$ there exists an invariable $C$ such that for any $\epsilon_{j} \leq 1$, $\delta_{i} \leq 1$ we have:

$$
\left|u^{\bar{\epsilon}, \overline{\delta^{\prime}}}-\left(u_{\overline{0}, \overline{0^{\prime}}}+\sum_{j=1}^{l} \sqrt{\epsilon_{j}} u_{\overline{1_{j}, \overline{0}^{\prime}}}+\sum_{i=1}^{n} \sqrt{\delta_{i}} u_{\overline{0}, \overline{1_{i}^{\prime}}}\right)\right| \leq C\left(\sum_{j=1}^{l} \epsilon_{j}+\sum_{i=1}^{r} \delta_{i}\right)
$$

Theorem 4 gives us information on how the approximate price behaves when $\epsilon_{j} \rightarrow 0$ and $\delta_{i} \rightarrow 0$.

Let $X$ be short interest rates. One of the most widely known models of short interest rates is the Vasicek model, in which $X$ is modeled as the Ornshtein-Ulenbeek process with multidimensional stochastic volatility. $\mathbb{P}$ dynamics of $X$ are given, in particular

$$
\begin{aligned}
d X_{t}= & \left(\kappa\left(\theta-X_{t}\right)-f\left(\mathrm{Y}_{1}, \ldots, \mathrm{Y}_{1}, \mathrm{Z}_{1}, \ldots, \mathrm{Z}_{\mathrm{r}}\right) \Omega\left(\mathrm{Y}_{1}, \ldots, \mathrm{Y}_{\mathrm{l}}, \mathrm{Z}_{1}, \ldots, \mathrm{Z}_{\mathrm{r}}\right)\right) d t \\
& +f\left(\mathrm{Y}_{1}, \ldots, \mathrm{Y}_{1}, \mathrm{Z}_{1}, \ldots, \mathrm{Z}_{\mathrm{r}}\right)+d \widetilde{W}_{t}^{x}, r\left(X_{t}\right)=X_{t}, h\left(X_{t}\right)=0,
\end{aligned}
$$

where $Y_{1}, \ldots, Y_{1}$, and $Z_{1}, \ldots, Z_{r}$ are fast and slowly variable volatility factors as described. We calculate the approximate price for a zero coupon bond. 
We write the operator $\left\langle\mathfrak{L}_{2}\right\rangle$ and the density associated with it at a rate $m(x)$

$$
\begin{gathered}
\left\langle\mathfrak{L}_{2}\right\rangle=\frac{1}{2} \bar{\sigma}^{2} \partial_{x x}^{2}+\kappa(\bar{\theta}-x) \partial_{x}-x, \\
m(x)=\frac{2}{\bar{\sigma}^{2}} \exp \left(\frac{-k}{\bar{\sigma}^{2}}(\bar{\theta}-x)^{2}\right), \quad \bar{\theta}=\theta-\frac{1}{\kappa} \overline{f \Omega}, \\
m(x)=\frac{2}{\bar{\sigma}^{2}} \exp \left(\frac{-k}{\bar{\sigma}^{2}}(\bar{\theta}-x)^{2}\right), \quad \bar{\theta}=\theta-\frac{1}{\kappa} \bar{f} \overline{ } .
\end{gathered}
$$

To find a bond price with a payoff $H\left(X_{t}\right)=\mathbb{I}_{\{\tau>t\}}=1$, we need to solve the equation (7) to find the eigenvalues for the segment $I=(-\infty, \infty)$ with $\left\langle\mathfrak{I}_{2}\right\rangle$ in compliance with $(8)$. Since both points $\infty$ and $\infty$ are natural limits, then the solution has the form [13].

$$
\begin{gathered}
\psi_{n}=\mathcal{N}_{n} \exp \left(-A \xi-\frac{1}{2} A^{2}\right) H_{n}(\xi+A), \\
\mathcal{N}_{n}=\left(\sqrt{\frac{\kappa}{\pi}} \frac{\bar{\sigma}}{2^{n+1} n !}\right)^{1 / 2}, \\
A=\frac{\bar{\sigma}}{\kappa^{3 / 2}}, \quad \xi=\frac{\sqrt{\kappa}}{\bar{\sigma}}(x-\bar{\theta}), \\
\lambda_{n}=\lambda_{n}=\bar{\theta}-\frac{\bar{\sigma}^{2}}{2 \kappa^{2}}+\kappa n, \quad n=0,1,2, \ldots .
\end{gathered}
$$

Here, $H_{n}$ are Hermite polynomials. We will write the expressions for the operators $\mathcal{A}_{j}$ and $\mathcal{B}_{j}$ :

$$
\mathcal{A}_{j}=-\vartheta_{j 3} \partial_{x x x}^{3}-\left(\vartheta_{j 2}+\mathfrak{U}_{j 2}\right) \partial_{x x}^{2}-\mathfrak{U}_{j 1} \partial_{x}, \quad \mathcal{B}_{j}=\vartheta_{j 1} \partial_{x}-\vartheta_{j 0}
$$

Operators $\mathcal{A}_{j k, n}, \mathcal{B}_{j k, n}$, and $\widetilde{\mathcal{B}}_{j k, n}$ are written on the basis of recurrence relations:

$$
\begin{aligned}
& \partial_{x} H_{n}=2 n H_{n-1}, \quad 2 x H_{n}=H_{n+1}+\partial_{x} H_{n}, \quad \mathcal{A}_{j k, n}=-\vartheta j_{3} \\
& \left\{\sum_{m=0}^{3 \wedge n}\left(\begin{array}{c}
3 \\
m
\end{array}\right)\left(\frac{-1}{\kappa}\right)^{3-m}\left(\frac{2 \sqrt{\kappa}}{\bar{\sigma}}\right)^{m} \frac{n ! \mathcal{N}_{n}}{(n-m) ! \mathcal{N}_{n-m}} \delta_{k, n-m}\right\} \\
& \left\{\sum_{m=0}^{3 \wedge n}\left(\begin{array}{c}
2 \\
m
\end{array}\right)\left(\frac{-1}{\kappa}\right)^{2-m}\left(\frac{2 \sqrt{\kappa}}{\bar{\sigma}}\right)^{m} \frac{n ! \mathcal{N}_{n}}{(n-m) ! \mathcal{N}_{n-m}} \delta_{k, n-m}\right\} \\
& -\mathfrak{U}_{j 1}\left\{\left(\frac{-1}{\kappa}\right) \delta_{k, n}+\left(\frac{2 \sqrt{\kappa}}{\bar{\sigma}}\right) \frac{n ! \mathcal{N}_{n}}{(n-1) ! \mathcal{N}_{n-1}} \delta_{k, n-1}\right\}, \\
& \mathcal{B}_{j k, n}=-\vartheta_{1}\left\{\left(\frac{-1}{\kappa}\right) \delta_{k, n}+\left(\frac{2 \sqrt{\kappa}}{\bar{\sigma}}\right) \frac{n ! \mathcal{N}_{n}}{(n-1) ! \mathcal{N}_{n-1}} \delta_{k, n-1}\right\}-\vartheta_{j 0} \delta_{k, n}, \\
& \widetilde{\mathcal{B}}_{j k, n}=-\vartheta_{j 1} \bar{\sigma}^{\prime}\left\{\left[\left(\frac{-1}{\kappa}\right)\left(\frac{1}{2 \bar{\sigma}}-\frac{\bar{\sigma}}{\kappa^{3}}-\frac{n}{\bar{\sigma}}\right)\right] \delta_{k, n}+\left[\left(\frac{-1}{\kappa}\right)\left(\frac{4}{\kappa^{\frac{3}{2}}}\right)+\left(\frac{2 \sqrt{\kappa}}{\bar{\sigma}}\right)\left(\frac{1}{2 \bar{\sigma}}-\frac{\bar{\sigma}}{\kappa^{3}}-\frac{n}{\bar{\sigma}}\right)\right] \frac{n ! \mathcal{N}_{n}}{(n-1) ! \mathcal{N}_{n-1}} \delta_{k, n-1}\right. \\
& \left.+\left[\left(\frac{-1}{\kappa}\right)\left(\frac{-2}{\bar{\sigma}}\right)+\left(\frac{2 \sqrt{\kappa}}{\bar{\sigma}}\right)\left(\frac{4}{\kappa^{\frac{3}{2}}}\right)\right] \frac{n ! \mathcal{N}_{n}}{(n-2) ! \mathcal{N}_{n-2}} \delta_{k, n-2}+\left[\left(\frac{2 \sqrt{\kappa}}{\bar{\sigma}}\right)\left(\frac{-2}{\bar{\sigma}}\right)\right] \frac{n ! \mathcal{N}_{n}}{(n-3) ! \mathcal{N}_{n-3}} \delta_{k, n-3}\right\} \\
& -\vartheta_{i 0} \bar{\sigma}^{\prime}\left\{\left(\frac{1}{2 \bar{\sigma}}-\frac{\bar{\sigma}}{\kappa^{3}}-\frac{n}{\bar{\sigma}}\right) \delta_{k, n}+\left(\frac{4}{\kappa^{\frac{3}{2}}}\right) \frac{n ! \mathcal{N}_{n}}{(n-1) ! \mathcal{N}_{n-1}} \delta_{k, n-1}+\left(\frac{-2}{\bar{\sigma}}\right) \frac{n ! \mathcal{N}_{n}}{(n-2) ! \mathcal{N}_{n-2}} \delta_{k, n-2}\right\} \\
& -\vartheta_{i 1} \overline{f \Omega}^{\prime}\left\{\left(\frac{1}{\kappa^{3}}\right) \delta_{k, n}+\left(\frac{-4}{\bar{\sigma}^{\frac{3}{2}}}\right) \frac{n ! \mathcal{N}_{n}}{(n-1) ! \mathcal{N}_{n-1}} \delta_{k, n-1}+\left(\frac{4}{\bar{\sigma}^{2}}\right) \frac{n ! \mathcal{N}_{n}}{(n-2) ! \mathcal{N}_{n-2}} \delta_{k, n-2}\right\} \\
& -\vartheta_{i 0} \overline{f \Omega^{\prime}}\left\{\left(\frac{-1}{\kappa^{2}}\right) \delta_{k, n}+\left(\frac{2}{\bar{\sigma} \sqrt{\kappa}}\right) \frac{n ! \mathcal{N}_{n}}{(n-1) ! \mathcal{N}_{n-1}} \delta_{k, n-1}\right\} .
\end{aligned}
$$

Calculation of $c_{n}$ can be found in [12]

$$
c_{n}=\left(\psi_{n}, 1\right)=\frac{2}{\bar{\sigma}} \sqrt{\frac{\pi}{\kappa}} \mathcal{N}_{n} A^{n} e^{-A^{2} / 4} .
$$

The approximate price of a bond can now be calculated applying the theorems 1-3. 
For zero-coupon bonds, the bond curve is often considered, and not the bond price itself. The yield $R^{\bar{\epsilon}} \overline{\delta^{\prime}}$ for a zero-coupon bond, on which one dollar is paid at time $t$ is defined by the relation:

$$
u^{\bar{\epsilon}, \overline{\delta^{\prime}}}=\exp \left(-R^{\bar{\epsilon}, \overline{\delta^{\prime}}} t\right)
$$

We obtain the approximation for a zero-coupon bond, developing in series as bond prices $u^{\bar{\epsilon} \overline{\delta^{\prime}}}$, and the yield $R^{\bar{\epsilon}} \overline{\delta^{\prime}}$ in orders $\sqrt{\epsilon_{j}}$ and $\sqrt{\delta_{i}}$ :

$$
\begin{gathered}
u_{\overline{0}, \overline{0^{\prime}}}+\sum_{j=1}^{l} \sqrt{\epsilon_{j}} u_{\overline{\overline{1}_{j}, \overline{0^{\prime}}}}+\sum_{i=1}^{r} \sqrt{\delta_{i}} u_{\overline{0}, \overline{1_{i}^{\prime}}}+\cdots=e^{-\left(R_{\overline{0}, \overline{0}^{\prime}}+\sum_{j=1}^{l} \sqrt{\epsilon_{j}} R_{\overline{1_{j}}, \overline{0}^{\prime}}+\sum_{i=1}^{n} \sqrt{\delta_{i}} R_{\overline{0}, \overline{1}_{i}^{\prime}}\right) t} \\
=e^{-R_{\overline{0}, \overline{0}^{\prime}} t}+\sum_{j=1}^{l} \sqrt{\epsilon_{j}} R_{\overline{1_{j}, 0^{\prime}}} e^{-R_{\overline{0}, \overline{0}^{\prime}} t}+\sum_{i=1}^{r} \sqrt{\delta_{i}} R_{\overline{0}, \overline{1_{i}^{\prime}}} e^{-R_{\overline{0}, \overline{0}^{\prime}} t}+\cdots
\end{gathered}
$$

Grouping in orders $\sqrt{\epsilon_{j}}$ and $\sqrt{\delta_{i}}$ we obtain:

$$
\begin{gathered}
R^{\bar{\epsilon}, \overline{\delta^{\prime}}} \approx R_{\overline{0}, \overline{0^{\prime}}}+\sum_{j=1}^{l} \sqrt{\epsilon_{j}} R_{\overline{1_{j}}, \overline{0^{\prime}}}+\sum_{i=1}^{r} \sqrt{\delta_{i}} R_{\overline{0}, \overline{1_{i}^{\prime}}}, \\
R_{\overline{0}, \overline{0^{\prime}}}=-\frac{1}{t} \ln \left(u_{\overline{0}, \overline{0^{\prime}}}\right), \quad R_{\overline{1_{j}, 0^{\prime}}}=\frac{-u_{\overline{1_{j}, 0^{\prime}}}}{t u_{\overline{0}, \overline{0^{\prime}}}}, \quad R_{\overline{0}, \overline{1_{i}^{\prime}}}=\frac{-u_{\overline{0}, \overline{1_{i}^{\prime}}}}{t u_{\overline{0}, \overline{0^{\prime}}}} .
\end{gathered}
$$

Note that figures are constructed component-wise on each corresponding time scale, in much the same way as components in [2], [11].

\section{CONCLUSIONS}

The spectral theory and the theory of singular and regular perturbations are applied to investigate the short-term interest rates described by the Vasicek model. The approximate price of bonds and their yield are calculated. Applying the Sturm-Liouville theory, Fredholm's alternatives, as well as analysing singular and regular perturbations in different time scales, we obtained explicit formulas for the convergence of bond prices and yields. To obtain explicit formulas, we need to solve 21 Poisson equations. The main advantage of our pricing methodology is that by combining methods from spectral theory, regular perturbation theory, and the theory of singular perturbations we reduce everything to the solution of the equations to find their eigenfunctions and eigenvalues.

Developed methodology for modeling the pricing of derivatives, makes it possible to predict the results of targeted actions of stock market participants, and to correct behavior based on the influence of various factors present in the market.

\section{REFERENCES}

[1] Borodin A., Salminen, P. Handbook of Brownian motion: facts and formulae. Birkhauser, 2002.

[2] Burtnyak I.V., Malytska A.P. CEV Model with Stochastic Volatility. Journal of Vasyl Stefanyk Precarpathian National University, 6 (3-4) (2019), 22-28. doi: 10.15330/jpnu.6.3-4.22-28

[3] Malytska H., Burtnyak I. Construction of the fundamental solution of a class of degenerate parabolic equations of high order. Carpathian Math. Publ., 12 (1) (2020), 79-87. doi:10.15330/cmp.12.1.79-87

[4] Burtnyak I.V., Malytska H.P. Degenerate parabolic systems of the diffusion type with inertia. Journal of Mathematical Sciences, 249 (3) (2020). doi: 10.1007/s10958-020-04947-2

[5] Cox J.C., Ingersoll J.E., Ross S.A. A theory of the term structure of interest rates. Econometrica, 53 (2) (1985), 385-408.

[6] Going-Jaeschke A., Yor M. A survey and some generalizations of Bessel processes. Bernoulli, 9 (2) (2003), 313-349. doi: 10.2307/3318942 
[7] Gorovoi V., Linetsky V. Black's model of interest rates as options, eigenfunction expansions and Japanese interest rates. Mathematical finance, 14 (1) 2004, 49-78. doi: 10.1111/j.0960-1627.2004.00181.x

[8] Ho T.S.Y., Lee S. Term Structure Movements and Pricing Interest Rate Contingent Claims. The Journal of Finance, 41 (5) (1986), 1011-1029. doi: 10.1111/j.1540-6261.1986.tb02528.x

[9] Hull J., White A. The pricing of options on assets with stochastic volatilities. The Journal of Finance, 42 (2) (1987), 281-300. doi: 10.1111/j.1540-6261.1987.tb02568.x

[10] Linetsky V. The spectral decomposition of the option value. International Journal of Theoretical and Applied Finance, 7 (3) (2004), 337-384. doi: 10.1142/S0219024904002451

[11] Lorig M.J. Pricing Derivatives on Multiscale Diffusions: an Eigenfunction Expansion Approach. Mathematical Finance, 24 (2) (2014), 331-363. doi: 10.1111/mafi.12007

[12] Merton R.C. Theory of Rational Option Pricing. Bell Journal of Economics and Management Science, 4 (1) (1973), 141-183.

[13] Vasicek O. An equilibrium characterization of the term structure. Journal of Financial Economics, 5 (2) (1977), 177-188.

Address: Ivan Burtnyak, Anna Malytska, Vasyl Stefanyk Precarpathian National University, 57 Shevchenko St., Ivano-Frankivsk, 76018 Ukraine.

E-mail: ivan.burtnyak@pnu.edu.ua, hanna.malytska@pnu.edu.ua

Received: September 6, 2020; revised: September 14, 2020.

Буртняк Іван, Малицька Ганна. Моделювання ціноутворення деривативів методами спектрального аналізу. Журнал Прикарпатського університету імені Василя Стефаника, 7 (3) (2020), 128-136.

У цій статті розширюється метод знаходження наближеної ціни для широкого класу похідних фінансових інструментів. Використовуючи спектральну теорію самоспряжених операторів у Гільбертовому просторі та хвильову теорію сингулярних та регулярних збурень, встановлено аналітичну формулу наближеної ціни активів. Розроблено методи розрахунку цін опціонів за допомогою інструментів спектрального аналізу, теорії сингулярних та регулярних хвиль у випадку швидких і повільних факторів. Поєднуючи методи із спектральної теорії сингулярних та регулярних збурень, можна знайти ціну похідних фінансових інструментів як розкдад за власними функціями. Розраховується приблизна вартість цінних паперів та їх норма прибутку. Застосовуючи теорію Штурма-Ліувілля, альтернативу Фредгольма та аналіз сингулярних та регулярних збурень у різних часових масштабах можна отримати явні формули вартості цінних паперів та їх дохідності на основі розкладу власних функцій та власних значень самоспряжених операторів, що використовують крайові задачі для сингулярних та регулярних збурень. Доведено теорему, за допомогою якої знаходяться оцінки наближеної ціни деривативів. Розроблено алгоритм обчислення наближеної ціни деривативів і точності оцінок, що дозволяе проводити аналіз та зробити запобіжні висновки i пропозиції, щоб мінімізувати ризики щодо ціноутворення деривативів, які виникають на фондовому ринку. Розроблено модель знаходження величини ціни деривативів, що відповідають динаміці фондового ринку та величини фінансових потоків. Така модель дозводяе знаходити ціни деривативів та їхню волатильність, а також звести до мінімуму спекулятивні зміни в ціноутворенні, здійснювати аналіз проходження процесів на фондовому ринку та робити конкретні кроки для покращення ситуації щодо оптимізації фінансових стратегій. Використана методика ціноутворення європейських опціонів на основі дослідження поведінки волатидьності та аналізу дохідності фінансових інструментів дозволяе збільшити точність прогнозу та приймати обгрунтовані управлінські стратегічні рішення учасниками фондового ринку.

Ключові слова: фондовий ринок, деривативи, спектральний аналіз, спектральна теорія, сингулярна теорія збурень, регулярна теорія збурень. 